\title{
Diseño del esquema de calidad de la experiencia turística en hoteles*
}

\author{
Recibido: 6 de febrero de 2020 - Aprobado: 30 de marzo de 2020 \\ https://doi.org/10.22395/seec.v23n54a6
}

\author{
Ivis Taide González Camejo** \\ Leudis Orlando Vega de la Cruz ${ }^{* *}$ \\ Juan Antonio Chong Poutou ${ }^{* * *}$
}

\section{RESUMEN}

La calidad de la experiencia turística constituye un elemento determinante en la satisfacción del cliente a partir de la última década. El objetivo de este artículo es diseñar el esquema de calidad de la experiencia turística para entidades hoteleras. Para ello, se emplearon herramientas estadísticas que facilitaron la identificación de los elementos determinantes, siguiendo el enfoque del ciclo de gestión. Como principal resultado de esta investigación, se presenta un esquema de calidad de la experiencia turística adaptado a las características del sector hotelero que integra los componentes, dimensiones y ámbitos de la experiencia que más influyen en la percepción de la calidad por parte del cliente, lo que permite identificar las expectativas del cliente y diseñar estrategias en función de estas.

\section{PALABRAS CLAVE}

Calidad de la experiencia; gestión hotelera; experiencia turística; componentes; dimensiones.

\section{CLASIFICACIÓN JEL}

L83 y Z32

\section{CONTENIDO}

Introducción; 1. Dimensiones de calidad de la experiencia turística en hoteles. Componentes y ámbitos de la experiencia; 2 . La calidad de la experiencia turística desde la perspectiva del ciudadano cosmopolita; 3. Relación entre la calidad de la experiencia turística y la satisfacción del cliente; 4. Propuesta del esquema de calidad de la experiencia turística para entidades hoteleras;

5. Conclusiones; Bibliografía.

- Artículo de investigación. Proyecto de gestión organizacional, ejecución desde el 2015. Línea de gestión turística, del doctorado Tecnología para la gestión de la calidad de la experiencia turísticas en hoteles, tema de investigación de los autores.

.* Ingeniera Industrial, Universidad de Holguín, Holguín, Cuba. Máster en Ingeniería Industrial, Universidad de Holguín, Holguín, Cuba. Profesora e investigadora, Departamento de Ingeniería Industrial, Facultad de Ciencias Empresariales y Administración, Universidad de Holguín, Holguín, Cuba. Correo electrónico: ivist91@nauta.cu

-.. Ingeniero Industrial, Universidad de Holguín, Holguín, Cuba. Máster en Matemática Aplicada e Informática para la Administración, Universidad de Holguín, Holguín, Cuba. Profesor e investigador, Departamento de Ingeniería Industrial, Facultad de Ciencias Empresariales y Administración, Universidad de Holguín, Holguín, Cuba. Correo electrónico: leovega@uho.edu.cu

.... Ingeniero Industrial, Universidad de Holguín, Holguín, Cuba. Máster en Ingeniería Industrial, Universidad de Holguín, Holguín, Cuba. Correo electrónico: ivist91@nauta.cu 


\title{
DESIGN OF THE QUALITY SCHEME OF THE HOTELS TOURIST EXPERIENCE
}

\begin{abstract}
The quality of the tourist experiences constitutes a determinant element in customer satisfaction since the last decade. The main goal of this article is to design a quality scheme of the tourist experience for hotel entities, in order to do so this study employed statistic tools that facilitated the identification of the determinant elements and did so following the management cycle approach. As the main result of this study, it presents a quality scheme of the tourist experience adapted to the characteristics of the hotel industry that integrates the components, dimension and realm of experiences that influences the most in the perception of the quality by the customer, which allows the identification of customer expectancies and the design of a strategy based on these.
\end{abstract}

\section{KEY WORDS}

Quality of experience; hotel management; tourist experience; components; dimensions.

\section{CLASSIFICATION JEL}

L83 y Z32

\section{CONTENT}

Introduction; 1 . The dimension of the quality of tourist experience in hotels. Components and realms of experience; 2 . The quality of the tourist experience from the cosmopolitan citizen perspective; 3. The relation between the quality of the tourist experience and customer satisfaction; 4. Proposal for a quality scheme of the tourist experience for hotels; 5 . Conclusions; Bibliography.

\section{DESENHO DO ESQUEMA DE QUALIDADE DA EXPERIÊNCIA TURÍSTICA EM HOTÉIS}

\section{RESUMO}

A qualidade da experiência turística constitui um elemento-chave na satisfação do cliente na última década. O objetivo deste artigo é desenhar o esquema de qualidade da experiência turística para entidades hoteleiras; para isso, foram utilizadas ferramentas estatísticas que facilitaram a identificação desses elementos, seguindo o enfoque do ciclo de gestão. Como principal resultado da pesquisa, é apresentado um esquema de qualidade da experiência turística adaptado às características do setor hoteleiro que integra os componentes, as dimensões e os contextos da experiência que mais influenciam na percepção da qualidade por parte do cliente, o que permite identificar as expectativas deste e elaborar estratégias em função delas.

\section{PALAVRAS-CHAVE}

Qualidade da experiência; gestão hoteleira; turismo de experiência; componentes; dimensões.

\section{CLASSIFICAÇÃO JEL}

\author{
L83 y Z32
}

\section{CONTEÚDO}

Introdução; 1. Dimensões da qualidade da experiência turística em hotéis. Componentes e âmbitos da experiência; 2. Qualidade da experiência turística sob a perspectiva do cidadão cosmopolita; 3. Relação entre a qualidade da experiência turística e a satisfação do cliente; 4. Proposta do esquema de qualidade da experiência turística para entidades hoteleiras; 5. Conclusões; Bibliografia. 


\section{INTRODUCCIÓN}

El turismo de experiencias alcanza, actualmente, más reconocimiento por su estrecho vínculo con las emociones de las personas, por lo cual investigadores como Perdue (2002, p. 24) y Kos, Lončarić y Dlačić (2016) relacionan directamente la satisfacción y el bienestar con el ocio como experiencia. Por tal motivo, se han diversificado las propuestas de gestión turística para contribuir a la experiencia. Las intenciones de los turistas se aproximan en gran medida a la necesidad de experimentar sensaciones diferentes, y estas experiencias se establecen como el auténtico beneficio de la industria turística actual, ya que la calidad funcional de los servicios no basta para lograr su plena satisfacción, por lo cual se ha convertido en una condición necesaria pero insuficiente para el deleite y la fidelización, según Carballo et al. (2015), Boné, Rey y Fusté (2015) y Sánchez et al. (2017).

Las experiencias gratas y, por tanto, razonablemente notables, deben ser imprevistas, fortuitas, y que capten la atención del turista. Desde una perspectiva humanístico-psicológica, Gândara, Brea y Manosso (2013) y Damián (2018) consideran que estas experiencias vinculan el desarrollo personal coligado con el bienestar concebido a partir de la interacción con otras personas por vínculos familiares, de amistad o a través del contacto con integrantes de las comunidades locales visitadas que proyectan un efecto de beneficio exterior; por tal motivo, resulta esencial el aporte del turismo de experiencias al progreso de las relaciones interculturales gratas y enriquecedoras en los turistas y las comunidades visitadas (Sáez Cala, 2009).

La gestión de la experiencia turística se orienta hacia las dimensiones cognitiva, afectiva y sostenible, como consecuencia de su evidente carácter humanista y subjetivo (Pinassi y Ercolani, 2015; Chim-Miki, Batista-Canino y Medina-Brito, 2016; Sutty Segovia y Cruz Cardentey, 2018; Barrios y Reis, 2019). Los ámbitos en los que se desarrolla la experiencia se asocian al entretenimiento, al aprendizaje, al disfrute estético y a la aventura, mientras que los componentes de la experiencia turística (actores, actividades, escenarios y tematización) deben articularse en cada una de las etapas del ciclo de gestión para garantizar la satisfacción de los clientes. Además, según plantean Minkiewicz, Evans y Bridson (2014), debe incorporarse la tecnología como componente determinante de la experiencia.

El objetivo de la presente investigación se enmarca en elaborar una propuesta de esquema de calidad de la experiencia turística para hoteles teniendo en cuenta las principales tendencias teórico-metodológicas sobre el tema. Entre los métodos empleados para la realización del presente trabajo se encuentran las técnicas para recopilar información, generar ideas y lograr consenso, además de las encuestas a clientes. Además, se utilizó el análisis estadístico de centralidad en la revisión 
teórica y el análisis de las redes sociales, el cual se refiere a un conjunto de conceptos, que identifica las propiedades que poseen los individuos (u otros elementos) en tanto que participantes en una red de relaciones, ya las pruebas estadísticas no paramétricas. La distribución subyacente de estas no se ajusta a los llamados criterios paramétricos ya que los datos observados determinan si no se puede asumir que los mismos se ajusten a una distribución conocida y si el nivel de medida empleado no es, como mínimo, de intervalo.

\section{DIMENSIONES DE CALIDAD DE LA EXPERIENCIA TURÍSTICA EN HOTELES. COMPONENTES Y ÁMBITOS DE LA EXPERIENCIA}

Las buenas prácticas en la gestión de la calidad de la experiencia turística (CET) son: implicar al turista vinculando los elementos del entorno con las características individuales y desarrollar estrategias de gestión basadas en el equilibrio positivo entre las expectativas y percepción de los visitantes. Para profundizar en los elementos asociados a la calidad de la experiencia y la forma en que se articulan para lograr satisfacer las expectativas del cliente, se realizó una revisión de la bibliografía especializada en el tema'. Para el análisis se tuvieron en cuenta los atributos asociados a la CET y las dimensiones en las que estos se agrupan; las investigaciones fueron clasificadas en tres grupos.

El primer grupo está compuesto por el 22,4 \% de los autores y comprende los atributos relacional, sensorial, emocional y cultural. El segundo grupo abarca el 25,2\% de los autores y aborda los atributos físico, sostenible, afectivo, responsable, racional, cognitivo, social y espiritual. El tercer grupo contiene al 52,4\% de los autores con los atributos: confort, estimulante, novedoso o escapista, hedonista, ecológico, diversión, autenticidad y renovador.

Una vez analizados los resultados se asume la gestión de la CET por medio de los veintiún atributos de calidad identificados, siendo estos los de mayor representatividad en las investigaciones consultadas, con porcentajes de referencia explícita superior al $43 \%$. Igualmente, resulta pertinente incorporar cada uno de estos atributos a las dimensiones cognitiva, afectiva y sostenible, ya que se encuentran reflejados en los tres grupos identificados. Estas dimensiones quedan definidas, en la literatura revisada, como elementos genéricos en los cuales pueden agruparse los atributos enunciados. Se identificaron ciertos atributos cuya inclusión, como dimensiones fundamentales, puede valorarse ya que el $54,2 \%$ de los trabajos expresa relaciones de pertenencia entre ellos y el 47,5 \% establece la clasificación

En total, fueron ciento siete artículos científicos publicados en redes académicas como Scielo y Scopus. 
de dimensión general. Para determinar los atributos de calidad más representativos se construyó una matriz binaria que consideró la presencia o no de los atributos en cada uno de las investigaciones analizadas.

Utilizando el paquete estadístico Statistic Program for Social Sciences (SPSS) para Windows versión 21.0 y el Borgatti, S.P., Everett, M.G. and Freeman, L.C. 2002. Ucinet for Windows: Software for Social Network Analysis. Harvard, MA: Analytic Technologies, se obtuvo que los atributos más relacionados en las investigaciones analizadas fueron el cognitivo, afectivo, sensorial, social y sostenible (ver gráfico 1). Igualmente, se realizó un análisis de forma cuantitativa con la ayuda del Ucinet, cuyos resultados se muestran en el anexo 3.

\section{Gráfico 1. Análisis de centralidad de las dimensiones de CET}

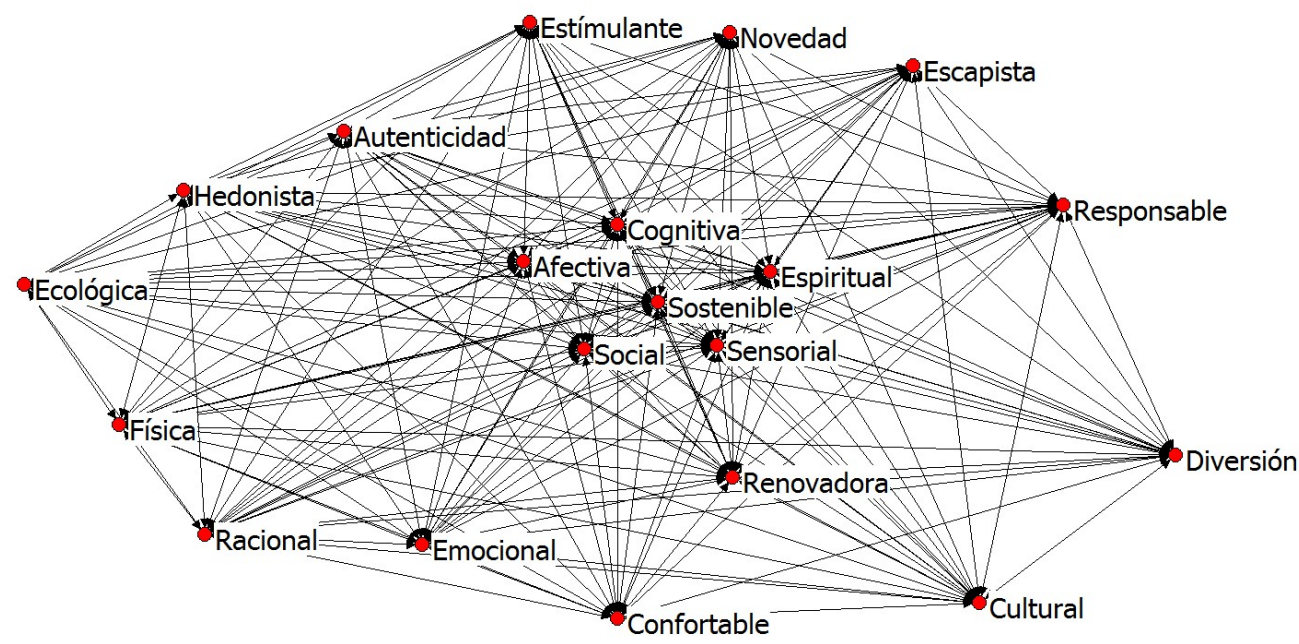

Fuente: elaboración propia.

En el gráfico se observa que los atributos anteriormente mencionados presentan un alto grado de centralidad normalizada con respecto a las restantes. De igual modo, tienen menor centralización global de cercanía con la red (in Closeness), mayor centralización global de la cercanía armónica (in Harmonic Closeness) y mayor robustez en los valores del eigenvector como medida de centralidad. Los atributos que presentan menor grado de centralidad son el racional, espiritual, cultural, responsable, físico, ecológico, diversión, autenticidad, relacional, renovadora, confortable, estimulante, emocional, hedonista, novedad y escapista. De forma general, puede decirse que todos los atributos deben ser evaluados y se considera necesario su análisis; 
no obstante, son los atributos de mayor centralidad los que serán considerados dimensiones de CET.

Una revisión más profunda permitió identificar las asociaciones de pertenencia que dan lugar a los criterios de agrupación por concepto del porcentaje de referencia explícita en las investigaciones. La dimensión cognitiva se asocia con los atributos: cultural (12,3\%), hedonista (11,9\%) y novedad (39,7 \%). La dimensión afectiva contiene los atributos: espiritual (21,3\%), estimulante (14,9\%), emocional $(62,3 \%)$ y escapista (33,8 \%). La dimensión sensorial contiene los atributos: físico $(44,9 \%)$, renovador (31,2 \%) y confortable (27,7 \%). La dimensión espiritual se asocia a los atributos cuyas combinaciones semánticas se relacionen con el atributo de diversión (74,3\%); mientras que la dimensión social concibe los atributos vinculados a la autenticidad (38,8\%). La dimensión sostenible se vincula a los atributos: racional (81,8 \%), responsable $(83,5 \%)$ y ecológico $(64,9 \%)$. Como parte de los parámetros analizados en la revisión bibliográfica, se tuvieron en cuenta los componentes que integran la experiencia turística y los ámbitos ${ }^{2}$ en que esta se desarrolla. Pudo concluirse que los componentes actividades ${ }^{3}$, escenarios ${ }^{4}$, actores ${ }^{5}$ y tematización ${ }^{6}$ son mencionados, al menos de forma explícita, en la totalidad de los trabajos. Además, se observa que el componente tecnología ${ }^{7}$ aparece en el 82,8 \% y corresponde, en su mayoría, a investigaciones realizadas a partir del año 2001. De igual modo se observa que la tematización aparece reflejada, además, como la principal forma de gestionar experiencias a nivel de producto como son: hoteles, cafeterías, restaurantes, excursiones y cruceros.

Todas las investigaciones desarrollan, al menos, un ámbito de la experiencia. Sin embargo, el entretenimiento (87,2 \%) constituye el más abordado seguido de aprendizaje (77,4\%), aventurero (71,7 \%) y estético (70,8\%). El 69,9\% de la literatura consultada trata los cuatro ámbitos de la experiencia de forma simultánea y el 42,6\% propone el ámbito escapista como una combinación de estos. De forma general, los componentes de la experiencia no se vinculan a las etapas de gestión, y la tecnología se aborda de forma aislada y sin vincularla al resto de los componentes definidos. La gestión de experiencias no es suficientemente investigada en hoteles, pese a que

2 Entiéndase como las temáticas o géneros de la experiencia turística: estético, aventurero, aprendizaje y entretenimiento.

3 Entiéndase como el conjunto de acciones, vinculadas a la proyección de experiencias, realizadas como parte de los servicios turísticos.

4 Entiéndase como el espacio físico donde se desarrollan las actividades, pueden ser diseñados, adaptados o aprovechados.

5 Entiéndase como los clientes y trabajadores (coactores) que participan de la experiencia.

6 Entiéndase como el diseño uniforme de las actividades en función de una temática determinada.

7 Entiéndase como la disposición de la infraestructura tecnológica en beneficio de la experiencia turística. 
estos clasifican entre los principales productos de un destino. La orientación hacia el cliente constituye el principal enfoque en materia de gestión de la calidad de experiencias turísticas, ningún trabajo consultado interrelaciona las seis dimensiones de calidad identificadas.

Los modelos consultados revelan que el tratamiento a la calidad de la experiencia turística se basa en el diagnóstico de las expectativas respecto a las dimensiones, en su mayoría en el orden cognitivo y afectivo; simultáneamente, estos se orientan hacia la predeterminación de los ámbitos y no conciben su predominio en las expectativas para proyectar los componentes. La tecnología se aborda como un recurso independiente. Aunque de forma teórica es aceptada como componente esencial, su mayor tratamiento se halla en investigaciones asociadas a experiencia en destinos turísticos inteligentes. El alcance de los modelos se orienta a la medición, evaluación y proyección de experiencias sin realizar un tratamiento diferenciado respecto a las oportunidades de mejora y el monitoreo continuo al desempeño de los elementos. Los indicadores se basan mayormente en al análisis cualitativo, lográndose una cuantificación elemental en el procesamiento de las expectativas e índices de satisfacción sin establecer parámetros para los componentes y ámbitos. Los instrumentos para la obtención de la información no establecen diferenciación específica entre la calidad experiencial y la funcional, y se basan en la percepción sobre las dimensiones. Sin embargo, los indicadores de análisis se limitan al tratamiento de las dimensiones, de actividades, actores y tematización, y el ámbito propicio según las características del destino y los productos. Los resultados obtenidos permiten afirmar la existencia de un cuerpo metodológico amplio y actualizado acerca de la gestión de experiencias turísticas y la gestión de la calidad, sin embargo, constituye un desafío integrar ambos constructos y elaborar herramientas adaptables a los ámbitos, componentes y dimensiones de calidad.

\section{LA CALIDAD DE LA EXPERIENCIA TURÍSTICA DESDE LA PERSPECTIVA DEL CIUDADANO COSMOPOLITA}

Existen criterios que abordan el consumo de experiencias turísticas desde la perspectiva del ciudadano global o cosmopolita ${ }^{8}$ (Archibugi, 2008; Augusto, 2003). No obstante, no se han encontrado evidencias empíricas que avalen la repercusión de estos elementos en la valoración que pueda tener determinado cliente respecto a la CET. Con el propósito de profundizar en el tema, se decidió aplicar una encuesta a un grupo de turistas (visitantes al destino Cuba en el año 2018) sobre elementos

Entiéndase como una persona que desea trascender la división geopolítica que es inherente a las ciudadanías nacionales de los diferentes Estados y países soberanos. 
asociados a la CET. En esta se incluyeron variables inherentes a la valoración de las dimensiones de CET y las expectativas respecto al hotel.

Se diseñó un plan de selección, se determinó el tamaño de la muestra según la expresión de cálculo para poblaciones infinitas y se seleccionó un muestreo aleatorio simple. Asumiendo un error admisible del $5 \%$, la cantidad de turistas a encuestar fue de trescientos ochenta y cuatro. Se extendió el muestreo en un 10 \% (treinta y ocho clientes) para cubrir la posibilidad de anulación de la encuesta. De un total de cuatrocientos veintitrés clientes encuestados, el 4,1 \% entregó la encuesta incompleta y el 2,6 \% se negó a participar en el estudio. Fueron procesadas trescientas noventa y cuatro encuestas cuyo resultado permitió determinar la fiabilidad de la escala (Alfa de Cronbach $=0,976$ ) y la validez del instrumento (Coeficiente $\mathrm{KMO}=0,941$ ), con la ayuda del paquete estadístico SPSS versión 21.0 para Windows. De un total de trescientas noventa y cuatro encuestados, doscientos once $(28,4 \%)$ pertenecen al sexo masculino y ciento ochenta y tres (71,6 \%) son mujeres. Los rangos etarios oscilaron entre 16 y 83 años. La procedencia predominante fue Canadá (39,6 \%), seguida por Inglaterra (22,1 \%), Estados Unidos (15,5\%), Cuba (11,2 \%), Francia (7,1 \%), Reino Unido (2,6 \%) y Alemania (1,9\%); el nivel cultural predominante fue medio (78,3\%). Para determinar la relación de las variables nacionalidad, sexo, nivel cultural y edad con la valoración sobre la CET se tuvo en cuenta la escala de evaluación y el tipo de variable.

Tanto la variable dependiente como las explicativas se dividieron en dos categorías. En el caso de la variable nacionalidad se analizaron los dos continentes en los cuales se clasifican los países encuestados (Europa y América). El nivel cultural se clasificó en no superior ${ }^{9}$ y superior ${ }^{10}$; y la edad en mayores y menores de 40 años. Utilizando el procesador estadístico SPSS versión 21.0 para Windows se realizó un análisis de regresión logística binaria. De la evaluación de los estadísticos descriptivos se concluyó que la mayor valoración sobre la ET la poseen los clientes menores de 40 años, del sexo masculino, graduados de nivel superior y procedentes del continente americano. La comparación de medias arrojó que la edad promedio de los clientes, con una valoración alta y baja sobre la ET, es de 32 y 31 años, respectivamente.

Podría decirse que la diferencia entre las variables independientes, respecto a la valoración, no es de gran magnitud y que, por la misma razón, no se esperaría que estas fueran significativas en el estudio. Simultáneamente, el análisis de regresión

\footnotetext{
9 Niveles previos al superior

10 Entiéndase como la última etapa del proceso de aprendizaje académico, es decir, a todas las trayectorias formativas posecundarias que cada país contempla en su sistema. Puede definirse como universitario.
} 
logística permite confirmar, mediante la prueba Chi-cuadrado $\left(X^{2} \exp =1,867\right.$, g.l. $=4$ $=\mathrm{n}^{\circ}$ de covariables; $\left.\mathrm{Sig} .=0,760>0,05\right)$, que ninguna de las variables independientes (edad, sexo, nacionalidad y nivel cultural) está asociada a la valoración sobre la ET (ver tablas 1-3). En este caso el análisis concluye sin necesidad de analizar las medidas de resumen del modelo (R Cuadrado de Cox y Snell y Nagelkerke) ni el ajuste de los datos (prueba de Hosmer y Lemeshow).

Tabla 1. Resumen del procesamiento de los casos

\begin{tabular}{llcc}
\hline \multicolumn{2}{c}{ Casos no ponderados } & N & Porcentaje \\
\hline \multirow{3}{*}{ Casos seleccionados } & Incluidos en el análisis & 394 & 100,0 \\
\cline { 2 - 4 } & Casos perdidos & 0 & 0 \\
\cline { 2 - 4 } & Total & 394 & 100,0 \\
\hline Casos no seleccionados & 0 & 0 \\
\hline Total & 394 & 100,0 \\
\hline
\end{tabular}

Fuente: elaboración propia a partir del paquete estadístico SPSS versión 21.0 para Windows.

Tabla 2. Codificaciones de variables categóricas

\begin{tabular}{llcc}
\hline & & Frecuencia & \multicolumn{1}{c}{$\begin{array}{c}\text { Codificación de } \\
\text { parámetros }\end{array}$} \\
\cline { 3 - 4 } Nivel cultural de los turistas & & No superior & \multicolumn{1}{c}{$(1)$} \\
\hline \multirow{2}{*}{ Sexo de los turistas } & Superior & 313 &, 000 \\
\hline \multirow{2}{*}{ Continente de procedencia de los turistas } & Masculino & 211 & 1,000 \\
\cline { 2 - 4 } & Femenino & 183 & 1,000 \\
\hline \multirow{2}{*}{ Edad de los turistas } & Europa & 261 &, 000 \\
\hline & Menores de 40 años & 281 & 1,000 \\
\cline { 2 - 4 } & 40 años o más & 113 & 1,000 \\
\hline
\end{tabular}

Fuente: elaboración propia a partir del paquete estadístico SPSS versión 21.0 para Windows.

Tabla 3. Pruebas ómnibus sobre los coeficientes del modelo

\begin{tabular}{llccc}
\hline & & Chi cuadrado & gl & Sig. \\
\hline \multirow{3}{*}{ Paso 1} & Paso & 1,867 & 4 &, 760 \\
\cline { 2 - 5 } & Bloque & 1,867 & 4 &, 760 \\
\cline { 2 - 5 } & Modelo & 1,867 & 4 &, 760 \\
\hline
\end{tabular}

Fuente: Elaboración propia a partir del Paquete estadístico SPSS versión 21.0 para Windows. 


\section{RELACIÓN ENTRE LA CALIDAD DE LA EXPERIENCIA TURÍSTICA Y LA SATISFACCIÓN DEL CLIENTE}

Las investigaciones consultadas durante la realización del presente trabajo exponen, al menos de forma implícita, que la satisfacción del cliente ha estado marcada por la calidad de la experiencia turística. Sin embargo, no evidencian resultados empíricos que midan objetivamente la relación entre las variables. Por tanto, se analiza la dependencia que pueda existir entre la CET y la satisfacción del cliente mediante el análisis del riesgo relativo o coeficiente de corrección tetracórica. Se tuvieron en cuenta los resultados de las preguntas correspondientes a cada variable en la encuesta. El objetivo esencial del análisis es identificar en qué medida la CET determina la satisfacción global del cliente. Para ello, se realizaron preguntas para evaluar la percepción sobre las dimensiones de calidad y preguntas asociadas a la satisfacción general respecto a los servicios. La escala de evaluación de ambas variables es dicotómica, las tablas 4 y 5 muestran los resultados del análisis.

Tabla 4. Pruebas de Chi-cuadrado

\begin{tabular}{lccccc}
\hline & Valor & gl & $\begin{array}{c}\text { Sig. asintótica } \\
\text { (bilateral) }\end{array}$ & $\begin{array}{c}\text { Sig. exacta } \\
\text { (6ilateral) }\end{array}$ & $\begin{array}{c}\text { Sig. exacta } \\
\text { (unilateral) }\end{array}$ \\
\hline Chi-cuadrado de Pearson & $292,902 a$ & 1 &, 000 & & \\
\hline Corrección por continuidad & 289,444 & 1 &, 000 & & \\
\hline Razón de verosimilitudes & 347,399 & 1 &, 000 & &, 000 \\
\hline Estadístico exacto de Fisher & & & & & \\
\hline Asociación lineal por lineal & 292,158 & 1 &, 000 & & \\
\hline N de casos válidos & 394 & & & & \\
\hline
\end{tabular}

Fuente: elaboración propia a partir del paquete estadístico SPSS versión 21.0 para Windows.

El resultado del estadístico corrección por continuidad (Sig. Exacta-bilateral; $0,00<0,005)$ conlleva a la conclusión de que, en función de la CET, la satisfacción del cliente será diferente.

Tabla 5. Estimación de riesgo

\begin{tabular}{lccc} 
& \multirow{2}{*}{ Valor } & \multicolumn{3}{c}{ Intervalo de confianza al 95\% } \\
\cline { 3 - 4 } & & Inferior & Superior \\
\hline $\begin{array}{l}\text { Razón de las ventajas para calidad de la experiencia turística } \\
\text { (alta/baja) }\end{array}$ & 183,857 & 84,086 & 402,009 \\
\hline Para la cohorte satisfacción del cliente $=$ alta & 12,266 & 7,406 & 20,316 \\
\hline Para la cohorte satisfacción del cliente $=$ baja &, 067 &, 039 &, 113 \\
\hline N de casos válidos & 394 & & \\
\hline
\end{tabular}

Fuente: elaboración propia a partir del paquete estadístico SPSS versión 21.0 para Windows. 
El riesgo relativo $(183,857)$ implica razón de las ventajas para la calidad de la experiencia turística (alta/baja). Por tanto se puede afirmar que el incremento de la CET genera un impacto positivo en la satisfacción del cliente.

\section{PROPUESTA DEL ESQUEMA DE CALIDAD DE LA EXPERIENCIA TURÍSTICA PARA ENTIDADES HOTELERAS}

El esquema que se propone se adapta a cualquier instalación hotelera y se concibe para los tres servicios básicos (alojamiento, alimentos y bebidas y animación) y sus actividades complementarias. Se estructura sobre la base de los fundamentos teóricos que integran la experiencia turística desde su contribución a la satisfacción del cliente. Esta herramienta puede ser empleada para la proyección de estrategias relacionadas con la CET y debe ser antecedida por un diagnóstico que valore el estado actual de los elementos incluidos, la misma puede segmentarse o ampliarse atendiendo a las características y necesidades del hotel. Para implementar el esquema deben haber sido determinadas las expectativas de los clientes y clasificadas según los ámbitos de la experiencia. Luego se construye la matriz de experiencia del hotel, tal y como aparece en la tabla 6.

\section{Tabla 6. Matriz de la experiencia turística para hoteles}

Ámbito predominante: (puede realizarse para cualquier ámbito o para aquel predominante en las expectativas)

\begin{tabular}{|c|c|c|c|c|c|}
\hline Expectativas & $\begin{array}{c}\text { Porcentaje de } \\
\text { clientes }\end{array}$ & Prioridad & Componentes & $\begin{array}{c}\text { Servicios del } \\
\text { hotel }\end{array}$ & $\begin{array}{l}\text { Dimensiones } y \\
\text { atributos de calidad }\end{array}$ \\
\hline \multirow{3}{*}{$\begin{array}{l}\text { Se listan las } \\
\text { expectativas } \\
\text { identificada }\end{array}$} & \multirow{3}{*}{$\begin{array}{l}\text { Porcentaje } \\
\text { de clientes } \\
\text { que posee la } \\
\text { expectativa }\end{array}$} & \multirow{3}{*}{$\begin{array}{l}\text { (Opcional) se } \\
\text { puede otor- } \\
\text { gar un orden } \\
\text { de prioridad }\end{array}$} & \multirow{3}{*}{$\begin{array}{l}\text { Componentes } \\
\text { de la experiencia } \\
\text { que se incluyen } \\
\text { dentro de la ex- } \\
\text { pectativa }\end{array}$} & Aloj. & \multirow{3}{*}{$\begin{array}{l}\text { Dimensiones y atri- } \\
\text { butos de calidad } \\
\text { que intervienen } \\
\text { (puede estar explí- } \\
\text { cito o implícito en } \\
\text { la expectativa) }\end{array}$} \\
\hline & & & & $A+B$ & \\
\hline & & & & Animación & \\
\hline
\end{tabular}

Fuente: elaboración propia.

Para construir esta matriz se recomienda apoyarse en un equipo de trabajo, ya que su estructura requiere de un análisis profundo a las expectativas definidas para establecer su interrelación con cada elemento de la matriz. Igualmente, se propone construir una matriz para cada ámbito y analizar las expectativas por ámbito. Para determinar la prioridad de las expectativas (opcional) se propone una escala, en función de la cantidad de clientes analizados (ver tabla 7). 
Tabla 7. Criterios para establecer el orden de prioridad de las expectativas

\begin{tabular}{cl}
\hline Clientes que comparten una expectativa (\%) & Prioridad \\
\hline $81-100$ & Prioridad 1 (muy importante) \\
\hline $50-80$ & Prioridad 2 (importante) \\
\hline $0-49$ & Prioridad 3 (menos importante) \\
\hline
\end{tabular}

Fuente: elaboración propia.

Debe tenerse en cuenta que el hecho de que el ámbito predominante ${ }^{11}$ sea uno, no implica que la experiencia no se proyecte en los restantes. Esto solo significa que las expectativas que dieron lugar a la definición de la experiencia tiene una orientación marcada hacia el ámbito predominante. Las técnicas que se pueden emplear son la revisión de comentarios en redes sociales, entrevistas, encuesta y métodos de consenso.

Para construir la matriz de experiencia del hotel se pueden calcular los índices de predominancia (IP) para los ámbitos según la expresión de cálculo y la escala que aparecen en la tabla 8. De esta forma se obtiene un criterio cuantificable de la presencia de cada ámbito en las expectativas de los clientes y a la vez una visión general de la experiencia que debe proyectar el hotel.

Tabla 8. Expresión de cálculo para los índices de predominancia (IP)

\begin{tabular}{cll}
\hline Índice de predominancia (IP) & \multicolumn{1}{c}{ Expresión de cálculo } & \multicolumn{1}{c}{ Leyenda } \\
\hline Ámbitos & $\mathrm{IPa}=\sum(\mathrm{V} / 100) /$ Expa & $\begin{array}{l}\text { IP: índice de predominancia de los ámbitos. } \\
\text { V: porcentaje de clientes que comparten las } \\
\text { expectativas asociadas al ámbito. } \\
\text { Expa: cantidad de expectativas por ámbito. }\end{array}$ \\
\hline Escala & $\begin{array}{l}\text { Se selecciona el (los) ma- } \\
\text { yor IP en una escala de 0-1. }\end{array}$ & $\begin{array}{l}\text { El IP predominante es el que debe poten- } \\
\text { ciarse en el diseño de la experiencia. }\end{array}$ \\
\hline
\end{tabular}

Fuente: elaboración propia.

\section{Técnicas: métodos de consenso y trabajo en grupo.}

Teniendo en cuenta que la forma en que se relacionan los componentes (actividades, escenarios, actores, tematización y tecnología) de la experiencia y las expectativas (intereses específicos del cliente sobre la experiencia turística) de los clientes constituye un elemento altamente subjetivo, se debe analizar de forma cualitativa. Para ello, se recomienda resumir la esencia de cada componente según los elementos

11 Ámbito (s) con mayor representatividad en las expectativas del cliente. 
reflejados en la matriz. De esta forma se podrán definir estrategias para cada componente, una vez se proyecte la experiencia, a través de métodos de consenso y trabajo en grupo. Una vez que se establecen estrategias concretas para cada una de las expectativas analizadas, en cada servicio del hotel, deben tenerse en cuenta los recursos necesarios para la implementación de cada estrategia y su prioridad (atendiendo al grado de importancia de la expectativa para el cliente). Para realizar el esquema de la experiencia se propone la tabla 9. Luego de elaborados los esquemas, se ponen las estrategias a consideración del consejo de dirección del hotel, este aprueba total o parcialmente las propuestas y se procede a su proyección.

Tabla 9. Esquema de la experiencia

\begin{tabular}{lc}
\hline $\begin{array}{l}\text { Estrategia (opcional, puede definirse de acuerdo a las expectativas conside- } \\
\text { radas, los recursos y elementos }\end{array}$ \\
\hline $\begin{array}{l}\text { Expectativas que satisface } \\
\text { Prioridad } \\
\text { (Tipo de prioridad) }\end{array}$ \\
\hline Servicio & Actividades (específicas para cada servicio) \\
\hline Alojamiento & \\
\hline Restauración & $\begin{array}{l}\text { Prioridad } \\
\text { Animación }\end{array}$ \\
\hline $\begin{array}{l}\text { Estrategia (opcional, puede definirse de acuerdo a las expectativas conside- } \\
\text { radas, los recursos y elementos implicados) }\end{array}$ \\
\hline $\begin{array}{l}\text { Expectativas que satisface } \\
\text { Servicio }\end{array}$ \\
\hline Alojamiento & Actividades (específicas para cada servicio) \\
\hline Restauración & \\
\hline Animación
\end{tabular}

Fuente: elaboración propia.

\section{CONCLUSIONES}

La gestión de la CET será efectiva en la medida que se logren diseñar, proyectar, controlar y mejorar los atributos y dimensiones de calidad identificados en cada uno de los componentes de la experiencia. Se realizó un examen bibliográfico basado en el estudio de las dimensiones que determinan la calidad de la experiencia turística utilizando un análisis de centralidad que permitió identificar los elementos determinantes, además de valorar la presencia de los ámbitos y componentes de la experiencia turística. Se determinó la relación entre las variables determinantes en 
la satisfacción del cliente y en la formación de la experiencia a través de pruebas no paramétricas. Se diseñó el esquema de CET para las entidades hoteleras y se proponen técnicas, como la matriz de la experiencia turística, respaldada por indicadores para establecer el orden de prioridad de las expectativas y el predominio de los ámbitos. De esta forma, se dota a las instalaciones hoteleras de instrumentos para la mejora de la calidad de sus servicios y la satisfacción de los clientes.

\section{BIBLIOGRAFÍA}

Archibugi, Daniele (2008). The global commonwealth of citizens: toward cosmopolitan democracy. Nueva Jersey: Princeton University Press, 320 p.

Augusto, Rafael (2003). Del cosmopolitismo a la globalización: Kant y la paz perpetua. En: Revista de Retórica y Teoría de la Comunicación, n. ${ }^{\circ}$ 5, p. 45-49.

Barrios, Yanet y Reis, Clóvis (2019). Autêntica Cuba: análise da publicidade turística de 2002-2016. En: Revista Brasileira de Pesquisa em Turismo, vol. 13, n. ${ }^{\circ}$ 1, p. 125-140. DOI: https://doi. $\overline{\text { org}} / 10.7784 /$ rbtur.v13i1.1497

Boné, Diana; Rey, Arantxa y Fusté, Francesc (2015). Los hoteles temáticos: un caso descriptivo de creación de experiencias a través de ejemplos singulares españoles. En: Papers de Turisme, n. ${ }^{\circ}$ 58, p. 47-57.

Carballo, Rita; Moreno-Gil, Sergio; León, Carmelo y Raymond, John (2015). Designing and promoting experiences in a tourist destination. An analysis of research and action needs. En: Cuadernos de Turismo, n. ${ }^{\circ}$ 35, p. 435-438.

Chim-Miki, Adriana; Batista-Canino, Rosa María y Medina-Brito, Pino (2016). Coopetición en un destino turístico de frontera entre Argentina, Brasil y Paraguay: el caso Poloiguassu. En: Semestre Económico, vol. 19, n. ${ }^{\circ} 40$, p. 145-174. DOI: https://dx.doi.org/10.22395/seec. $\overline{\mathrm{v} 19 \mathrm{n} 40 \mathrm{a} 6}$

Damián, Alfonso (2018). Construcción colectiva de la experiencia turística para la sociedad anfitriona, una mirada desde el sur. En: Revista Latino-Americana de Turismología, vol. 4, n. ${ }^{\circ}$ 1, p. 34-51. DOI: 10.34019/2448-198X.2018.v4.10044

Gândara, José Manoel; Brea, José Antonio y Manosso, Franciele Cristina (2013). Calidad de la experiencia en los hoteles termales de Galicia, España: un análisis a través de la reputación online. En: Estudios y Perspectivas en Turismo, vol. 22, n. 3, p. 492-525.

Kos, Andrijana; Lončarić, Dina y Dlačić, Jasmina (2016). Augmented reality experiential marketing in tourism. https://bit.ly/2zJBrmv

Minkiewicz, Joanna; Evans, Jody y Bridson, Kerrie (2014). How do consumers co-create their experiences? An exploration in the heritage sector. En: Journal of Marketing Management, vol. 30, n. ${ }^{\circ} 1-2$, p. 30-59. DOI: https://doi.org/10.1080/0267257X.2013.800899

Perdue, Richard (2002). Internet site evaluations. The influence of behavioral experience, existing images, and selected website characteristics. En: Journal of Travel and Tourism Marketing, vol. 11, n. ${ }^{\circ} 2-3$, p. 21-38. DOI: https://doi.org/10.1300/J073v11n02_02 
Pinassi, Andrés y Ercolani, Patricia (2015). Geografía del turismo: análisis de las publicaciones científicas en revistas turísticas. El caso de Argentina. En: Cuadernos de Geografía, vol. 24, n. ${ }^{\circ} 1$, p. $214-230$.

Sáez Cala, Antonia (2009). Los micro-clusters turísticos: ¿una herramienta para el desarrollo turístico en zonas rurales deprimidas? En: Semestre Económico, vol. 12, n. 24, p. 57-68.

Sánchez, Driselda; Maldonado, Minerva; Martínez, Luis y Lara, Graciela (2017). Rentabilidad de hoteles boutique explicada desde la experiencia memorable. En: El Periplo Sustentable, n. ${ }^{\circ} 33$, p. $81-104$.

Sutty Segovia, Hernán Isidro y Cruz Cardentey, Francisco (2018). Luque: potencialidades y perspectivas para el desarrollo del turismo cultural. En: Academo, vol. 5, n. ${ }^{\circ}$ 1, p. 3-12. 


\section{ANEXO 1.}

Instrumento para profundizar en el impacto de la satisfacción con la calidad de la experiencia turística en hoteles

Por favor con el propósito de garantizarle una experiencia turística memorable y a la altura de sus expectativas, le solicitamos responda las preguntas siguientes teniendo en cuenta que sus respuestas serán analizadas solamente con fines investigativos:

Datos generales: Edad Sexo Nacionalidad

1. Por favor, valore en una escala $1-5$ ( 1 es no valorado y 5 muy valorado) la medida en que considera importante durante su estancia en el hotel:

Valoración

Aprender cosas nuevas

Experimentar emociones memorables

Interactuar con las personas del hotel y la comunidad

Participar en actividades que contribuyan al desarrollo local y mantener una conducta responsable hacia el medio ambiente

Vincularse, con todos sus sentidos, en actividades que capten su total atención

2. Empleando la misma escala de la pregunta 1 (1 es muy bajo y 5 muy alto), diga cómo son sus expectativas y su percepción respecto al hotel, en cuanto a:

Expectativas Percepción

Aprender cosas nuevas

Experimentar emociones memorables

Interactuar con las personas del hotel y la comunidad

Participar en actividades que contribuyan al desarrollo local y mantener una conducta responsable hacia el medio ambiente

Vincularse, con todos sus sentidos, en actividades que capten su total atención.

3. Exprese, en una escala de 1-5 donde (1 es totalmente insatisfecho y 5 muy satisfecho), el grado de satisfacción respecto a la experiencia vivida en el hotel

4. ¿Cómo valora la calidad de la experiencia turística en el hotel de forma general?

5. Exprese, en una escala de 1-5 donde (1 es totalmente insatisfecho y 5 muy satisfecho), el grado de satisfacción respecto al hotel

6. ¿ ¿Recomendaría usted el hotel por la experiencia vivida durante su estancia en él? $\mathrm{Si} \_$No

Muchas gracias

Fuente: elaboración propia. 